\title{
Inertial Control of a DFIG-based Wind Power Plant using the Maximum Rate of Change of Frequency and the Frequency Deviation
}

\author{
Hyewon Lee*, Jinho Kim*, Don Hur** and Yong Cheol Kang ${ }^{\dagger}$
}

\begin{abstract}
In order to let a wind generator (WG) support the frequency control of a power system, a conventional inertial control algorithm using the rate of change of frequency (ROCOF) and frequency deviation loops was suggested. The ROCOF loop is prevailing at the initial stage of the disturbance, but the contribution becomes smaller as time goes on. Moreover, its contribution becomes negative after the frequency rebound. This paper proposes an inertial control algorithm of a wind power plant (WPP) using the maximum ROCOF and frequency deviation loops. The proposed algorithm replaces the ROCOF loop in the conventional inertial control algorithm with the maximum ROCOF loop to retain the maximum value of the ROCOF and eliminate the negative effect after the frequency rebound. The algorithm releases more kinetic energy both before and after the frequency rebound and increases the frequency nadir more than the conventional ROCOF and frequency loops. The performance of the algorithm was investigated under various wind conditions in a model system, which includes a doubly-fed induction generator-based WPP using an EMTP-RV simulator. The results indicate that the algorithm can improve the frequency drop for a disturbance by releasing more kinetic energy.
\end{abstract}

Keywords: Inertial control, Frequency deviation, Maximum rate of change of frequency and frequency support

\section{Introduction}

Wind power penetration in power systems has been globally increasing due to technical advances that have made the technology economically viable over the last decade. For example, the EU has set a goal of supplying $100 \%$ of electrical energy by renewable energy sources by 2050 and half of this energy will be supplied by wind power generation [1].

In order to keep the frequency within the acceptable ranges in a conventional power system, synchronous generators (SGs) with spinning reserves inherently respond to the frequency excursion and restore the reduced frequency to the nominal value by using the reserves.

On the other hand, variable-speed wind generators (VSWGs) have been widely used to extract maximum energy from wind $[2,3]$. To do this, VSWGs operate at a maximum power point tracking (MPPT) control mode, where they adjust the rotor speed depending on the wind speed. However, the MPPT control decouples the wind generators (WGs) from the system frequency and thus causes a reduction in the system inertia. Therefore, the

$\dagger$ Corresponding Author: Dept. of Electrical Engineering, WeGAT Research Center, and Smart Grid Research Center, Chonbuk National University, Korea. (yckang@jbnu.ac.kr)

* Dept. of Electrical Engineering and WeGAT Research Center, Chonbuk National University, Korea. (\{hyewonlee, jkim\}@jbnu.ac.kr)

** Dept. of Electrical Engineering, Kwangwoon University, Korea. (dhur@kw.ac.kr)

Received: August 18, 2014; Accepted: October 27, 2014 reliability of a power system will be jeopardized, particularly in the case of high wind penetration.

The frequency support scheme of the WG can be divided into two groups: inertial control and primary control. If a disturbance occurs, the former uses only the kinetic energy stored in the rotating masses, whilst the latter uses both the kinetic energy and the reserve power that is de-loaded. This paper addresses only the inertial control of a WG and thus it is assumed that the WGs are operating in the MPPT control mode before a disturbance.

A significant amount of research on the inertial control scheme has been proposed for the doubly-fed induction generators (DFIGs) [4-8]. An inertial control loop was designed using the rate of change of the frequency (ROCOF) $[4,5]$. The ROCOF loop generates additional power during the initial period of the disturbance and helps increase the frequency nadir. However, incremental power from the ROCOF loop decreases as time goes on. Moreover, the contribution of the ROCOF loop becomes negative after the frequency rebound. Thus, both the ROCOF and frequency deviation loops were used to improve the frequency support of DFIGs [6-8]. The frequency deviation loop generates an additional power reference proportional to the frequency deviation, and thus mitigates the negative power reference from the ROCOF loop after the frequency nadir.

This paper proposes an inertial control algorithm of a DFIG-based WPP using the maximum ROCOF and the frequency deviation loops. The proposed algorithm replaces 
the ROCOF loop in the conventional inertial control algorithm with the maximum ROCOF loop to retain the maximum value of the ROCOF and eliminate the negative effect after the frequency rebound. Thus, the algorithm releases more kinetic energy both before and after the frequency rebound and increases the frequency nadir more than the conventional inertial control algorithm. The performance of the algorithm is investigated with a model system, which consists of five SGs and a 100 MW WPP with 20 DFIGs using an EMTP-RV simulator. The wind speed of each DFIG was calculated considering the cumulative impact of multiple shadowing and the effect of the wind direction.

\section{Proposed Inertial Control of a DFIG-based WPP}

This paper proposes an inertial control algorithm of a WPP, which consists of multiple DFIGs. The following two subsections will describe the conventional and proposed inertial algorithms that will be implemented in each DFIG. Then, the wake model used in this paper will be explained.

\subsection{Conventional inertial control of a DFIG [6-8]}

Fig. 1 shows a conventional inertial controller of a DFIG that uses the ROCOF and frequency deviation loops. In the top loop of Fig. 1, $\Delta P_{\text {in }}$ depends on the ROCOF with $K$, which is the gain of the ROCOF loop, i.e.,

$$
\Delta P_{i n}=-K f_{s y s} \frac{d f_{s y s}}{d t}
$$

On the other hand, in the bottom loop of Fig. 1, $\Delta P$ depends on the frequency deviation with $R$, which is the droop gain of the loop, i.e.,

$$
\Delta P=-\frac{1}{R}\left(f_{s y s}-f_{\text {nom }}\right)
$$

The relationship between the mechanical power, $P_{m}$, and

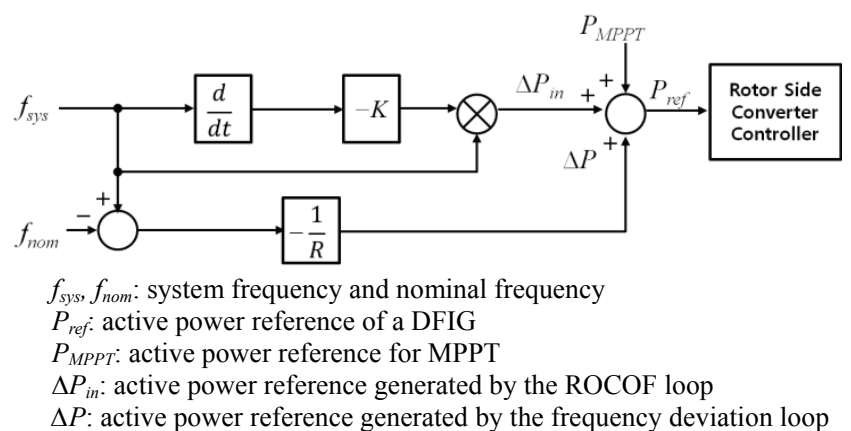

Fig. 1. Conventional inertial controller
$P_{\text {ref }}$ of a DFIG can be represented by

$$
P_{m}-P_{e}=P_{m}-\left(P_{M P P T}+\Delta P+\Delta P_{i n}\right)=J \omega_{r} \frac{d \omega_{r}}{d t}
$$

where $J$ is the moment of inertia $\left(\mathrm{kg} \cdot \mathrm{m}^{2}\right)$ of the rotor and $\omega_{r}$ is the rotor speed of the $\mathrm{WG}$.

If the system frequency drops below its nominal value for a disturbance such as a generator tripping, $\Delta P_{\text {in }}$ and $\Delta P$ become positive values, and consequently $P_{\text {ref }}$ increases. Thus, the kinetic energy stored in the rotating mass in the DFIG is released and consequently $\omega_{r}$ decreases according to (3).

At the initial stage of the disturbance, the contribution of the ROCOF loop becomes dominant, because the ROCOF has a large value and so does $\Delta P_{i n}$. As the frequency decreases further, $\Delta P_{\text {in }}$ decreases. In addition, $\Delta P_{\text {in }}$

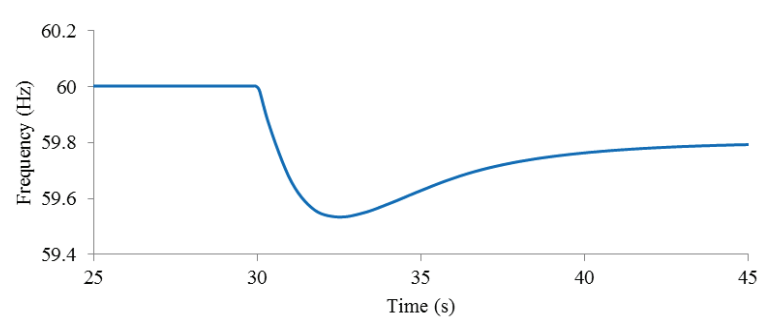

(a) System frequency

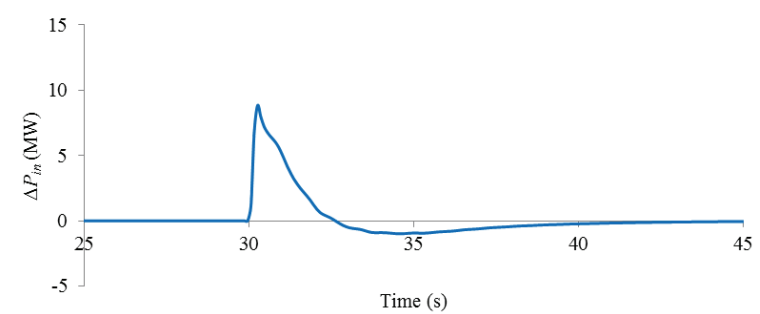

(b) $\Delta P_{\text {in }}$

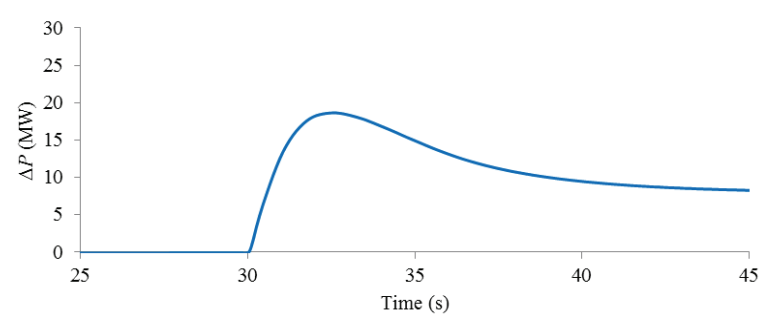

(c) $\Delta P$

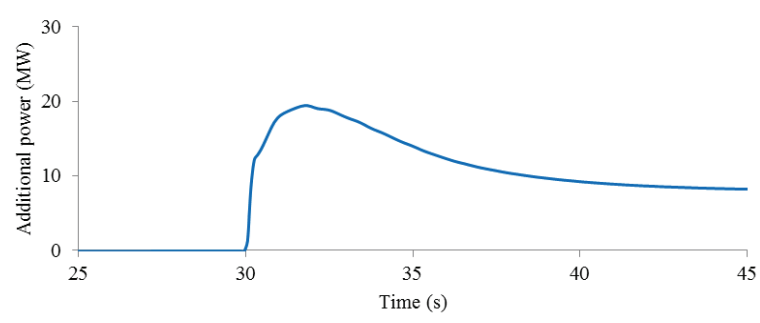

(d) Additional power of the supplementary loops

Fig. 2. Results for the conventional algorithm 
becomes a negative value after the frequency rebound. This has a negative impact on the inertial control of a DFIG.

To clearly illustrate the performance of the conventional algorithm, a test result is shown in Fig. 2, where a disturbance occurs at $30 \mathrm{~s}$. As mentioned above, $\Delta P_{\text {in }}$ decreases with time and becomes a negative value after the frequency rebound. On the other hand, $\Delta P$ remains a positive value. The additional power of the supplementary loops for the conventional algorithm helps increase the frequency nadir.

\subsection{Proposed inertial control of a DFIG}

In order to provide a greater contribution to frequency support, this paper replaces the ROCOF loop in the conventional controller in Fig. 1 with the maximum ROCOF loop, but leaves the frequency deviation loops intact. Fig. 3 shows the proposed inertial controller. The maximum ROCOF loop calculates the ROCOF, selects the maximum value of the previous ROCOF values, and then multiplies $-K$ to obtain $\Delta P_{i n, \max }$. Thus, $\Delta P_{i n, \max }$ remains a positive constant value with time after a short time, whereas $\Delta P_{\text {in }}$ in Fig. 1 decreases with time and becomes a negative value after the frequency rebound. Consequently, the proposed inertial controller can arrest the frequency nadir more effectively than the conventional controller.

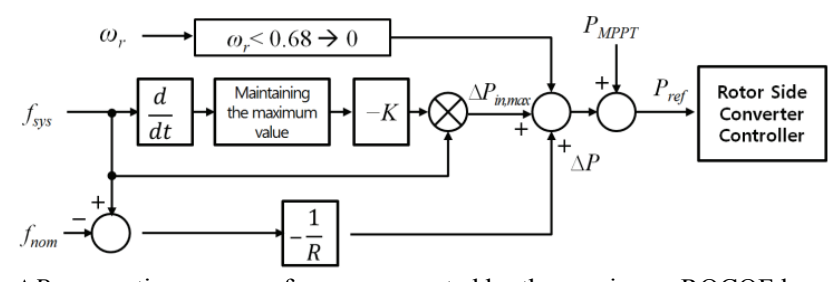

$\Delta P_{i n, \max }$ : active power reference generated by the maximum ROCOF loop

Fig. 3. Proposed inertial controller of a DFIG

On the other hand, the proposed algorithm releases a large amount of kinetic energy stored in the rotating mass and thus $\omega_{r}$ might decrease below the minimum operating speed. Typically, the operating range of the rotor speed is 0.68-1.32 pu. To prevent the rotor speed from decreasing below $0.68 \mathrm{pu}$, the additional control loops are disabled if $\omega_{r}$ reaches $0.68 \mathrm{pu}$.

Fig. 4 shows a test result for the proposed algorithm. Note that $\Delta P_{i n, \max }$ remains a positive constant value with time whilst $\Delta P_{i n}$ in Fig. 2(b) decreases with time and becomes a negative value after the frequency rebound. $\Delta P$ in Fig. 4(c) is similar to that in Fig. 2(c). Thus, the additional power of the supplementary loops for the proposed algorithm is larger than the conventional algorithm. Therefore, the proposed algorithm can improve the frequency nadir more than the conventional algorithm.

\subsection{Wake effect}

The WGs in a WPP generate electricity by extracting the

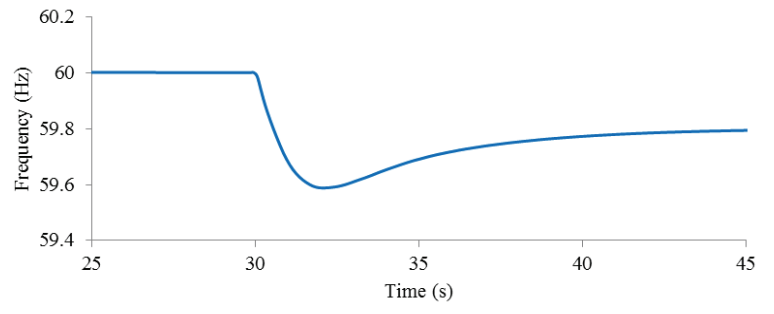

(a) System frequency

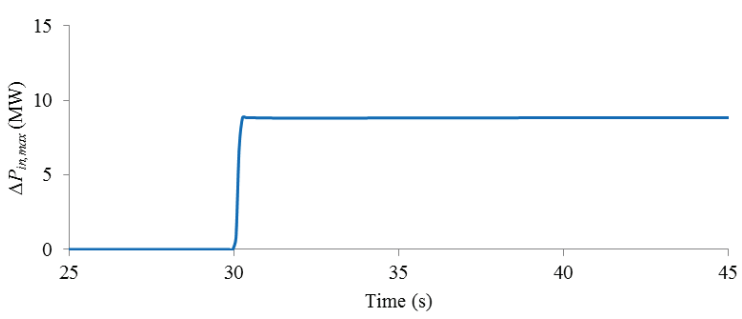

(b) $\Delta P_{\text {in, } \max }$

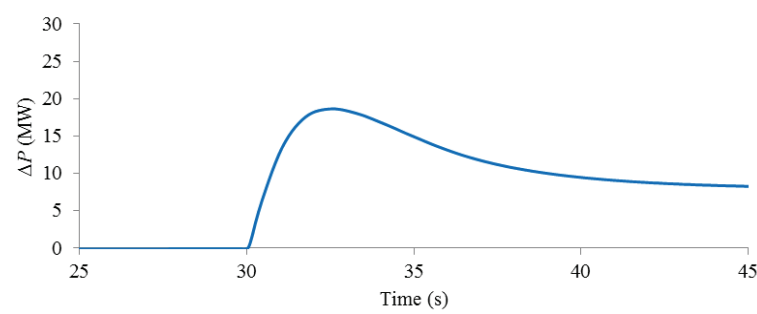

(c) $\Delta P$

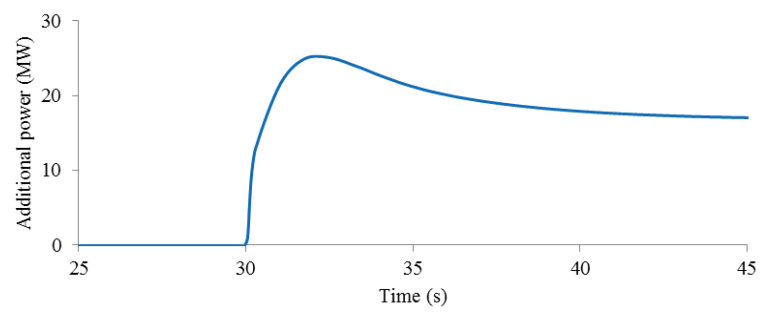

(d) Additional power of the supplementary loops

Fig. 4. Results for the proposed algorithm

kinetic energy from wind. Thus, the upstream WGs will impact the wind speed approaching at the downstream WGs. This shadowing effect is known as the wake effect [9]. Therefore, the different wind speeds approaching the WGs will result in different operating conditions such as the rotor speed and the power production of WGs.

In this paper, the wind speeds of WGs are calculated by considering the cumulative impact of multiple shadowing and the effect of the wind direction. The wind speed of a $\mathrm{WG}_{j}, v_{j}$, can be represented by

$$
v_{j}=v_{j 0}-\sqrt{\sum_{\substack{k=1 \\ k \neq j}}^{n} \beta_{k}\left\{v_{w k}\left(x_{k j}\right)-v_{j 0}\right\}^{2}}
$$

where $v_{j 0}$ is the incoming wind speed at $\mathrm{WG}_{j}$ without any shadowing, $x_{k j}$ is the radial distance between $\mathrm{WG}_{k}$ and $\mathrm{WG}_{j}$, 
$v_{w k}\left(x_{k j}\right)$ is the speed of the wind arriving at $\mathrm{WG}_{j}$ from the shadowing, $\mathrm{WG}_{k}, \beta_{k}$ is the ratio of part of the area of $\mathrm{WG}_{j}$ under the shadow of $\mathrm{WG}_{k}$ to its total area, and $n$ is the total number of WGs.

\section{Model system}

Fig. 5 shows a model system used to investigate the performance of the proposed inertial control algorithm. The model system consists of five SGs with a total of $850 \mathrm{MVA}$, a load of $600 \mathrm{MW}$ and 9 MVAr, and a 100 MW DFIGbased WPP. The detailed information of these will be described in the following subsections.

\subsection{Synchronous generators}

The five SGs connect to the model system and consist of one 100 MVA SG, one 150 MVA SG, and three 200 MVA SGs. As the governor model of SGs, an IEEEG1 model is used (see Fig. 6) and the parameters are shown in Table 1. The droop coefficient, $R$, of each $\mathrm{SG}$ is set to $5 \%$.

\subsection{Wind power plant}

As shown in Fig. 5, the WPP consists of five feeders, and four $5 \mathrm{MW}$ DFIGs are connected to each feeder. The distance between the neighboring WGs is $9 D(1,080 \mathrm{~m})$, where $D$ is the diameter of the swept area of the blades.

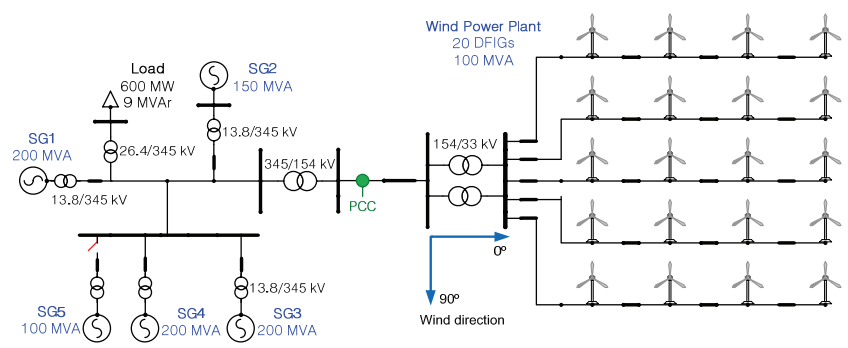

Fig. 5. Model system

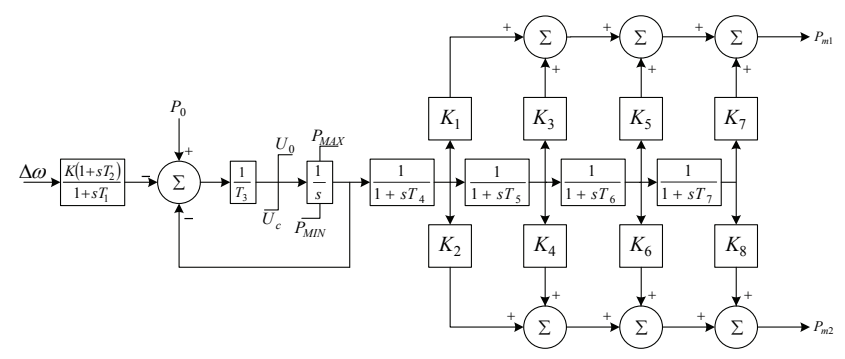

Fig. 6. IEEEG1 steam governor model

Table 1. Coefficients of the IEEEG1 model

\begin{tabular}{c|c|c|c|c|c|c|c|c|c}
\hline $\mathrm{K}$ & $\mathrm{T}_{1}$ & $\mathrm{~T}_{2}$ & $\mathrm{~T}_{3}$ & $\mathrm{U}_{\mathrm{o}}$ & $\mathrm{U}_{\mathrm{c}}$ & $\mathrm{P}_{\mathrm{MAX}}$ & $\mathrm{P}_{\text {MIN }}$ & $\mathrm{T}_{4}$ & $\mathrm{~K}_{1}$ \\
\hline 20 & 0.1 & 0 & 0.25 & 0.3 & -0.5 & 1 & 0.33 & 0.3 & 0.3 \\
\hline $\mathrm{K}_{2}$ & $\mathrm{~T}_{5}$ & $\mathrm{~K}_{3}$ & $\mathrm{~K}_{4}$ & $\mathrm{~T}_{6}$ & $\mathrm{~K}_{5}$ & $\mathrm{~K}_{6}$ & $\mathrm{~T}_{7}$ & $\mathrm{~K}_{7}$ & $\mathrm{~K}_{8}$ \\
\hline 0 & 10 & 0.4 & 0 & 0.4 & 0.3 & 0 & 0 & 0 & 0 \\
\hline
\end{tabular}

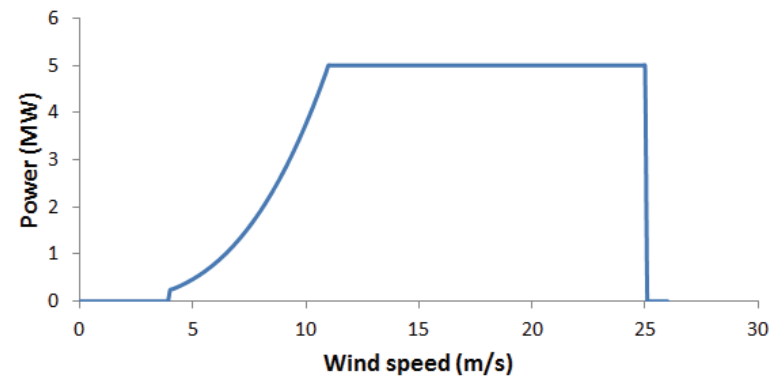

Fig. 7. Power curve of the WG

The two 60 MVA substation transformers are connected to the on-shore grid through a $22 \mathrm{~km}$-long submarine cable.

Fig. 7 shows the power curve of a DFIG, where the cutin, rated, and cut-out wind speed are $4 \mathrm{~m} / \mathrm{s}, 10.5 \mathrm{~m} / \mathrm{s}$, and $25 \mathrm{~m} / \mathrm{s}$, respectively. $P_{M P P T}$ for the MPPT control (as in [10]) was set to

$$
P_{M P P T}=k_{g} \omega_{r}^{3}
$$

where $k_{g}$ is a function of the parameters, such as the gearratio, blade length, and blade profile. In this paper, the operating range for the rotor speed of the DFIG is set to the range of $0.68-1.32 \mathrm{pu}$ for reliable operation.

\section{Case studies}

To simulate the frequency drop case, SG5 is assumed to be tripped out at $t=30 \mathrm{~s}$; it supplies $70 \mathrm{MW}$ to the load in the steady state. In this paper, the $R$ and $K$ gains of the auxiliary loops are set to 0.1 and 10 , respectively. Three cases are chosen and Table 2 shows the wind speeds of the WGs for the three cases calculated using (4). The performance of the proposed algorithm is compared with that of 'without (w/o) inertial control' and the conventional algorithm. In this paper, the supplementary loops of both the conventional and proposed algorithms are disabled if the rotor speed reaches $0.68 \mathrm{pu}$.

Table 2. Wind speeds of WGs for three cases considering the wake effect

\begin{tabular}{c|c|c|c|c|c|c|c|c|c|c|c}
\hline \multicolumn{4}{c|}{ Case 1 } & \multicolumn{6}{c|}{ Case 2 } & \multicolumn{4}{c}{ Case 3 } \\
\hline 8.25 & 7.38 & 6.60 & 5.91 & 10.31 & 9.23 & 8.25 & 7.39 & 10.31 & 10.31 & 10.31 & 10.31 \\
\hline 8.25 & 7.38 & 6.60 & 5.91 & 10.31 & 9.23 & 8.25 & 7.39 & 9.23 & 9.23 & 9.23 & 9.23 \\
\hline 8.25 & 7.38 & 6.60 & 5.91 & 10.31 & 9.23 & 8.25 & 7.39 & 8.25 & 8.25 & 8.25 & 8.25 \\
\hline 8.25 & 7.38 & 6.60 & 5.91 & 10.31 & 9.23 & 8.25 & 7.39 & 7.39 & 7.39 & 7.39 & 7.39 \\
\hline 8.25 & 7.38 & 6.60 & 5.91 & 10.31 & 9.23 & 8.25 & 7.39 & 6.60 & 6.60 & 6.60 & 6.60 \\
\hline
\end{tabular}

\subsection{Case 1: Wind speed of $8.25 \mathrm{~m} / \mathrm{s}$, wind direction of $0^{\circ}$}

Fig. 8 shows the results for case 1. Fig. 8(a) shows the system frequencies. The frequency nadirs for 'w/o inertial control', the conventional, and the proposed algorithms are $59.28 \mathrm{~Hz}, 59.48 \mathrm{~Hz}$, and $59.52 \mathrm{~Hz}$, respectively. The 


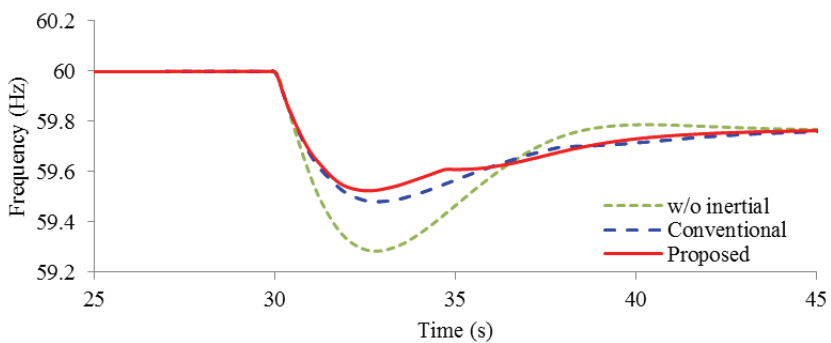

(a) System frequencies

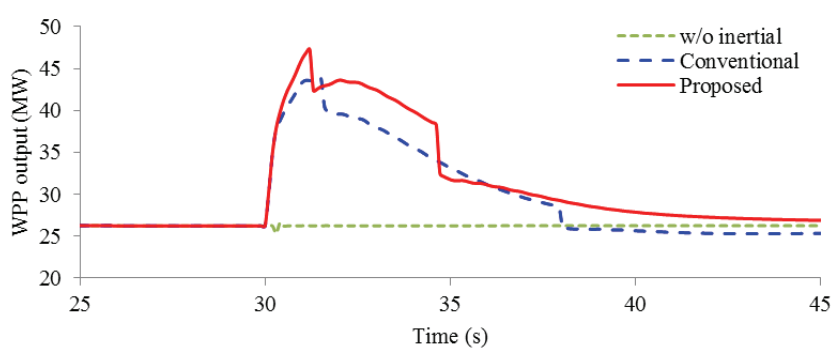

(b) Active power of the WPP

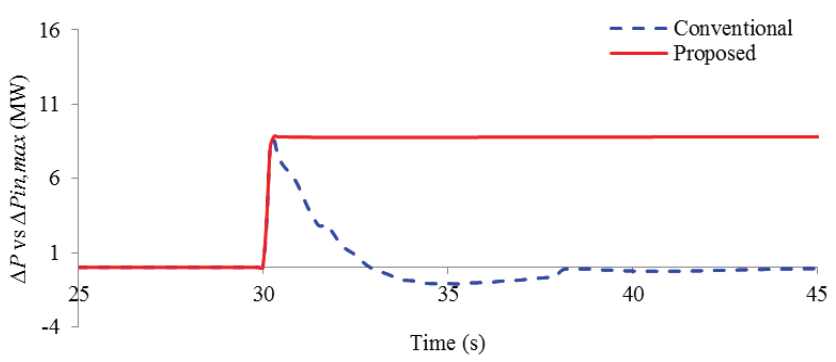

(c) $\Delta P_{\text {in }}$ and $\Delta P_{\text {in, } \max }$

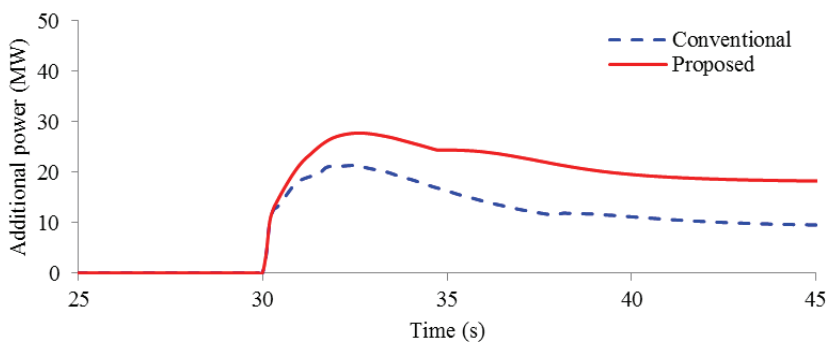

(d) Additional power of the supplementary loops

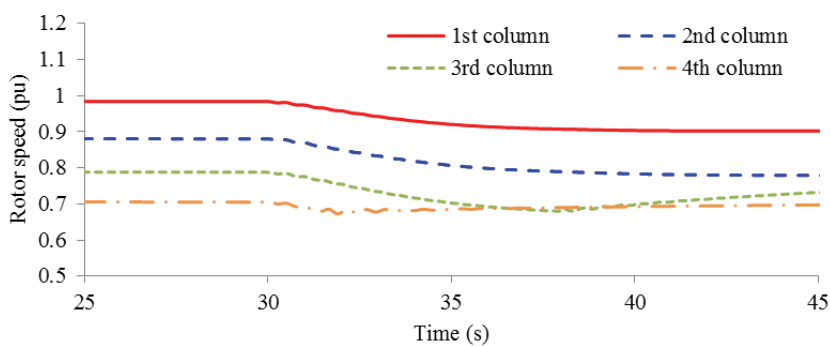

(e) Rotor speeds for the conventional algorithm

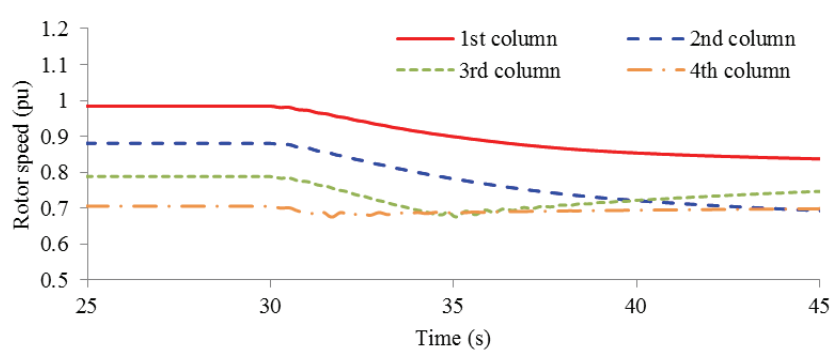

(f) Rotor speeds for the proposed algorithm

Fig. 8. Results for case 1

frequency nadir for the proposed algorithm is the largest because the former releases the most kinetic energy. From the view point of the nadir-based frequency response (NBFR), the NBFRs of ' $\mathrm{w} / \mathrm{o}$ inertial control', the conventional, and the proposed algorithms are $9.77 \mathrm{MW} / 0.1$ $\mathrm{Hz}, 13.48 \mathrm{MW} / 0.1 \mathrm{~Hz}$, and $14.70 \mathrm{MW} / 0.1 \mathrm{~Hz}$, respectively.

Fig. 8(b) shows the active power of the WPP. The maximum values of the active power for the conventional and proposed algorithms are 43.91 MW and 47.28 MW, respectively. The active power for the proposed algorithm is larger than that of the conventional algorithm because the additional power of the supplementary loops for the proposed algorithm is larger than that of the conventional algorithm, as shown in Figs. 8(c) and 8(d).

Fig. 8(c) shows $\Delta P_{i n, \max }$ and $\Delta P_{i n}$. As expected, $\Delta P_{i n, \max }$ remains constant with time, whereas $\Delta P_{\text {in }}$ decreases with time. The additional power of the supplementary loops for the proposed algorithm is larger than that for the conventional algorithm as shown in Fig. 8(d).

Figs. $8(\mathrm{e})$ and $8(\mathrm{f})$ show the rotor speeds of the four columns for the conventional and proposed algorithms, respectively. For the proposed algorithm, the rotor speeds of the WGs at only the third and fourth columns reach 0.68 pu at $31.59 \mathrm{~s}$ and $34.61 \mathrm{~s}$, respectively, whereas for the conventional algorithm, the rotor speeds at the third and fourth columns reach $0.68 \mathrm{pu}$ at $31.80 \mathrm{~s}$ and $37.93 \mathrm{~s}$, respectively. This is because the input wind speeds of the third and fourth columns are smaller than the first and second columns due to the wake effect. This will cause a sudden decrease in the active power of the WPP twice, as shown in Fig. 8(b).

\subsection{Case 2: Wind speed of $10.31 \mathrm{~m} / \mathrm{s}$, wind direction of $0^{\circ}$}

Fig. 9 shows the results for case 2, which is identical to case 1 except for the wind speed. As shown in Fig. 9(a), the frequency nadirs for 'w/o inertial control', the conventional, and the proposed algorithms are $59.31 \mathrm{~Hz}, 59.53 \mathrm{~Hz}$, and $59.59 \mathrm{~Hz}$, respectively. Similar to case 1 , the frequency nadir for the proposed algorithm is the largest. The NBFRs for 'w/o inertial control', the conventional, and the proposed algorithms are 10.22 MW/0.1 Hz, 15.01 MW/0.1 $\mathrm{Hz}$, and $17.00 \mathrm{MW} / 0.1 \mathrm{~Hz}$, respectively.

As shown in Fig. 9(b), the maximum values of the active power for the conventional and proposed algorithms are 


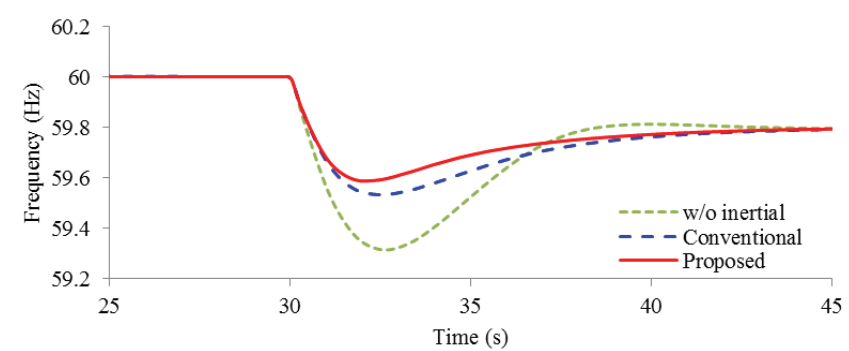

(a) System frequencies

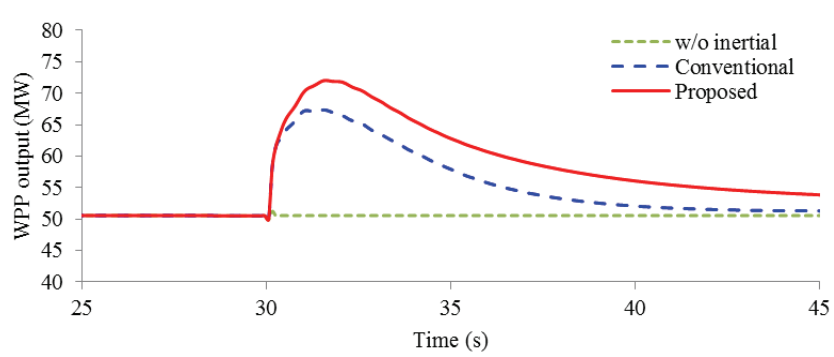

(b) Active power of the WPP

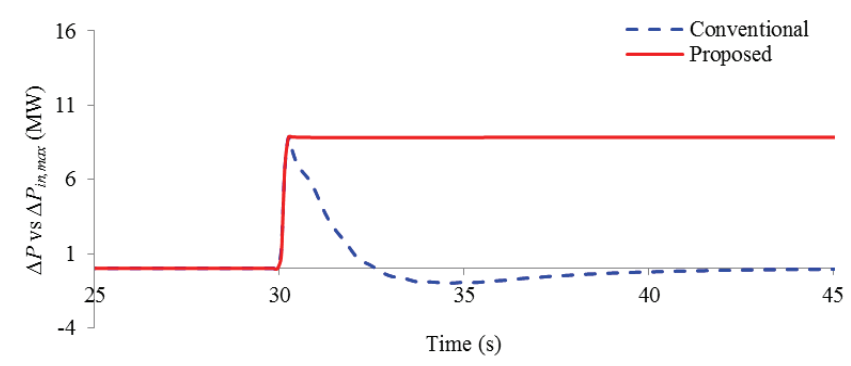

(c) $\Delta P_{\text {in }}$ and $\Delta P_{\text {in, } \max }$

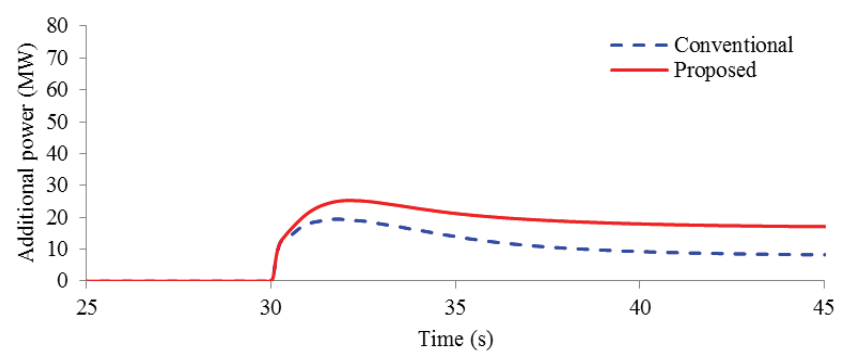

(d) Additional power of the supplementary loops

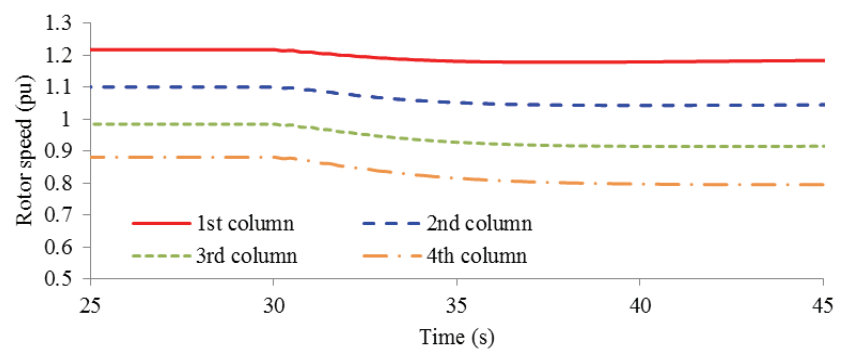

(e) Rotor speeds for the conventional algorithm

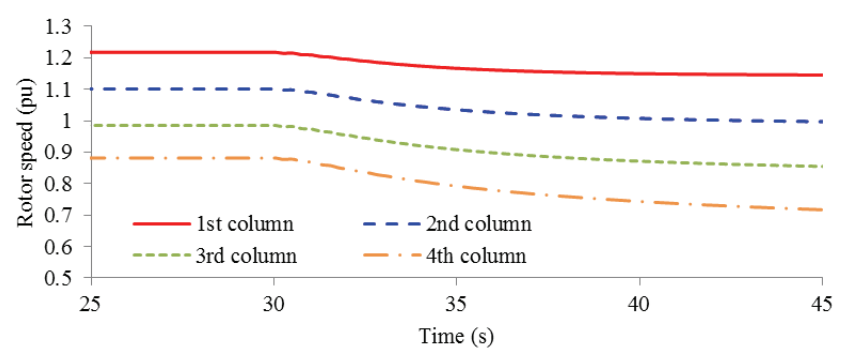

(f) Rotor speeds for the proposed algorithm

Fig. 9. Results for case 2

67.36 MW and 72.02 MW, respectively. The active power of the WPP for the proposed algorithm is larger than the conventional algorithm. Fig. $9(\mathrm{c})$ shows $\Delta P_{i n, \max }$ and $\Delta P_{i n}$. As in case $1, \Delta P_{i n, \max }$ remains a positive value with time whereas $\Delta P_{i n}$ decreases with time. As shown in Fig. 9(d), the additional power of the supplementary loops for the proposed algorithm is larger than that of the conventional algorithm during the disturbance.

The rotor speeds of the four columns for the conventional and proposed algorithms are shown in Figs. 9(e) and $9(f)$, respectively. The rotor speeds of the four columns remain larger than $0.68 \mathrm{pu}$ because the wind speed in case 2 is higher than in case 1 .

\subsection{Case 3: Wind speed of $10.31 \mathrm{~m} / \mathrm{s}$, wind direction of $90^{\circ}$}

Fig. 10 shows the results for case 3, which is identical to case 2 except for the wind direction. As shown in Fig. 10 (a), the frequency nadirs for 'w/o inertial control', the conventional, and the proposed algorithms are $59.29 \mathrm{~Hz}$, $59.51 \mathrm{~Hz}$, and $59.57 \mathrm{~Hz}$, respectively. The frequency nadir for the proposed algorithm is the largest because the released kinetic energy is the largest. The NBFRs for 'w/o inertial control', the conventional, and the proposed algorithms are 9.79 MW/0.1 Hz, 14.42 MW/0.1 Hz, and 16.32 MW/0.1 Hz, respectively. Note that the frequency nadirs and NBFR of the inertial control algorithm in case 3 are slightly smaller than in case 2 because the wake effect in case 3 is larger than that in case 2 due to the different wind direction.

The maximum values of the active power of the WPP for the conventional and proposed algorithms are 62.26 MW and 66.90 MW, respectively. As in the previous two cases, the additional power of the supplementary loops for the proposed algorithm is larger than that of the conventional algorithm. As shown in Fig. 10(d), the additional power of the supplementary loops for the proposed algorithm is larger than that of the conventional algorithm.

The rotor speeds of the five rows for the conventional and proposed algorithms are shown in Figs. 10(e) and 10(f), respectively. The rotor speeds of the WGs at only the fifth row reach $0.68 \mathrm{pu}$ at $35.14 \mathrm{~s}$ whereas the rotor speeds for the conventional algorithm do not reach $0.68 \mathrm{pu}$. This is because the input wind speeds of the WGs at the fifth row are lower than the other rows due to the severe wake effect 


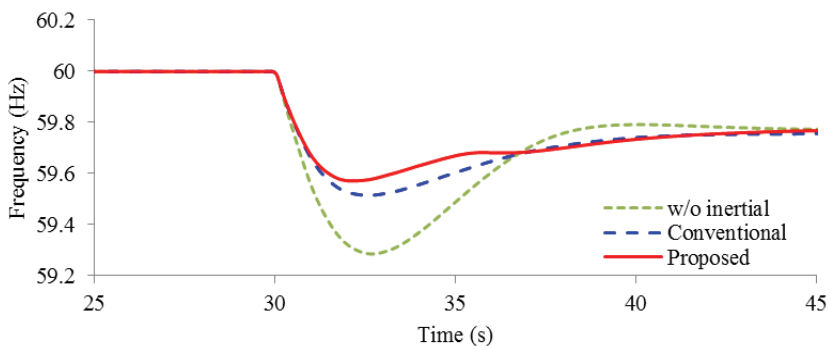

(a) System frequencies

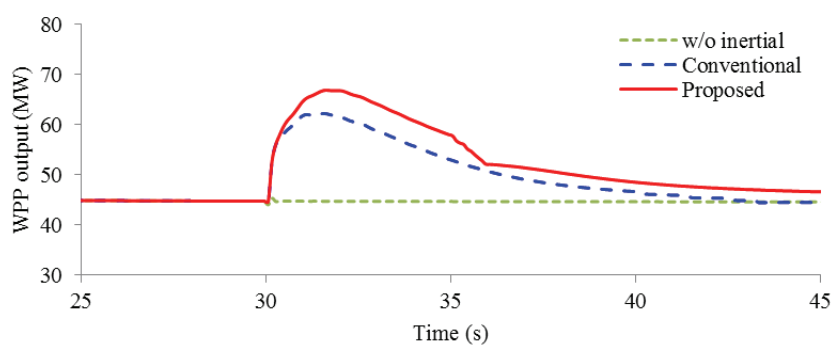

(b) Active power of the WPP

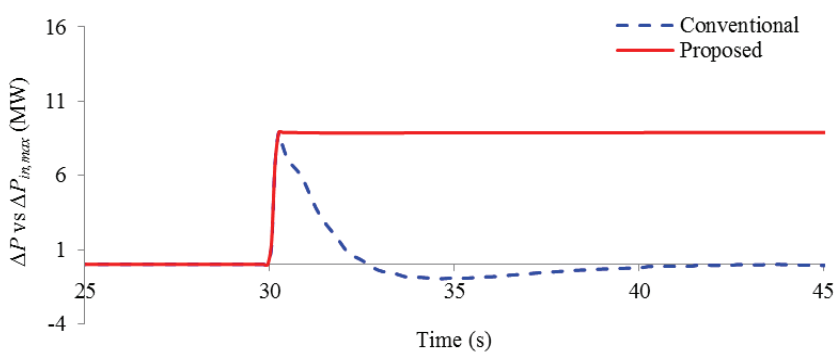

(c) $\Delta \mathrm{P}_{\text {in }}$ and $\Delta \mathrm{P}_{\text {in, max }}$

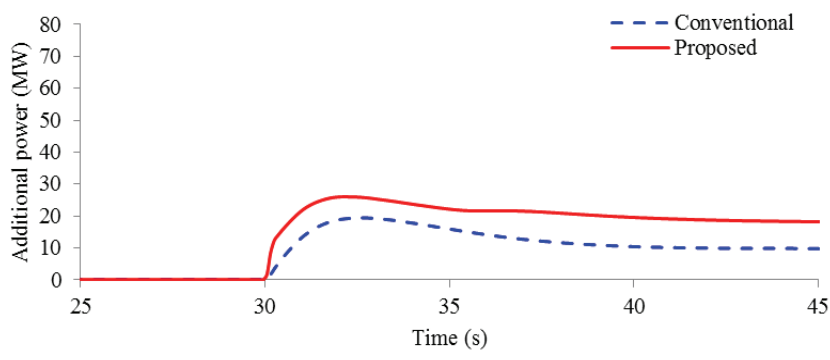

(d) Additional power of the supplementary loops

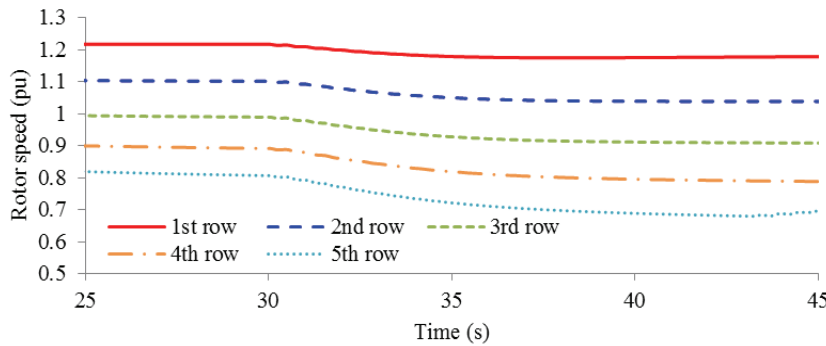

(e) Rotor speeds for the conventional algorithm

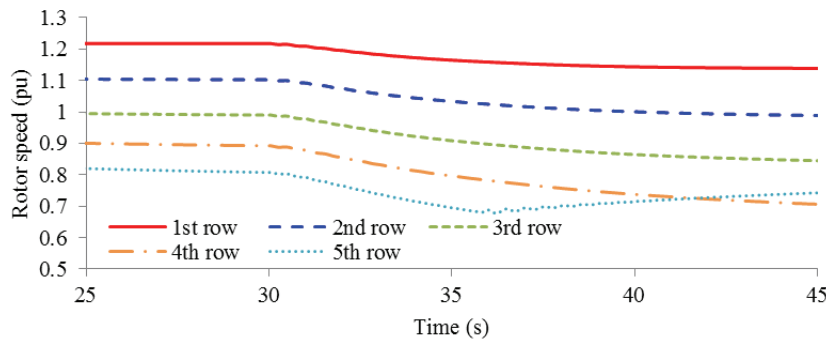

(f) Rotor speeds for the proposed algorithm

Fig. 10. Results for case 3

in this case (the $90^{\circ}$ wind direction). Thus, the active power of the WPP suddenly decreases at once, as shown in Fig. 10(b).

The results of the three cases clearly indicate that the inertial control algorithms can give more contribution if the WPP have more kinetic energy in the rotating masses.

\section{Conclusion}

This paper proposes an inertial control algorithm of a WPP using the maximum ROCOF and frequency deviation loops. The algorithm replaces the ROCOF loop in the conventional inertial control algorithm with the maximum ROCOF loop to retain the maximum value of the ROCOF and eliminate the negative effect after the frequency rebound. Thus, the algorithm can release more kinetic energy both before and after the frequency rebound and increase the frequency nadir more than the conventional inertial control algorithm.

The test results clearly show that the proposed algorithm is able to increase the frequency nadir by releasing more kinetic energy than a conventional algorithm and make the frequency rebound faster than the conventional algorithm.

\section{Acknowledgements}

This work was supported partly by the New \& Renewable Energy of the Korea Institute of Energy Technology Evaluation and Planning (KETEP) grant funded by the Korea government Ministry of Trade, industry \& Energy (2011T100200064) and partly by the National Research Foundation of Korea (NRF) grant funded by the Korea government (MSIP) (No.2010-0028509).

\section{References}

[1] Global Wind Energy Outlook 2012, GWEC, November 2012 .

[2] T. Ackermann, Wind Power in Power System, 2nd Edition, England, John Wiley \& Sons, Ltd, 2012.

[3] O. Anaya-lara, N. Jenkins, J. Ekanayake, P. Cartwright, and M. Hughes, Wind Energy Generation Modeling and Control, John Wiley \& Sons, Ltd, 2009. 
[4] J. B. Ekanayake, L. Holdsworth, and N. Jenkins, "Control of doubly fed induction generator wind turbine," IET Power Eng., vol. 17, no. 1, 2003, pp. 2832.

[5] J. Ekanayake and N. Jenkins, "Comparison of the response of doubly fed and fixed-speed induction generator wind turbines to changes in network frequency," IEEE Transaction on Energy conversion, vol. 19, no. 4, 2004, pp. 800-802.

[6] J. Morren, S. Haan, W. L. Kling, and J. A. Ferreira, "Wind turbines emulating inertia and supporting primary frequency control," IEEE Transaction on Power systems, vol. 21, no. 1, 2006, pp. 433-434.

[7] G. Ramtharan, J. B. Ekanayake, and N. Jenkins, "Frequency support from doubly fed induction generator wind turbines," IET Renew. Power Gen., vol. 1, 2007, pp. 3-9.

[8] Z. S. Zhang, Y. Z. Sun, J. Lin, and G. J. Li, "Coordinated frequency regulation by doubly fed induction generator-based wind power plants," IET Renew. Power Gener., vol. 6, no. 1, 2012, pp. 38-47.

[9] F. Koch, M. Gresch, F. Shewarega, I. Erlich, and U. Bachmann, "Consideration of wind farm wake effect in power system dynamic simulation," in Proc. IEEE Power Tech. Conf., June 2005, pp. 1-7.

[10] B. Shen, B. Mwinyiwiwa, Y. Zhang, and B. Ooi, "Sensorless Maximum Power Point Tracking of Wind by DFIG Using Rotor Position Phase Lock Loop," IEEE Transaction on Power Electronics, vol. 24, no. 4, 2009, pp. 942-951.

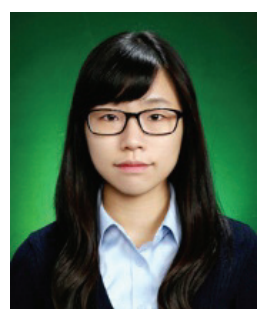

Hyewon Lee She received the B.S. degree from Chonbuk National University, Korea in 2010. She is studying for her Ph.D. degree at Chonbuk National University and is also an assistant researcher at the Wind energy Grid-Adaptive Technology (WeGAT) Research Center supported by the Ministry of Science, ICT, and Future Planning (MSIP), Korea. Her research interests include the development of wind energy grid integration techniques.

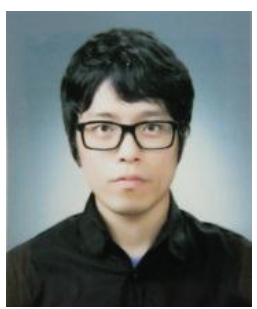

Jinho Kim He received his B.S. degree from Chonbuk National University, Korea in 2013. He is currently pursuing his M.S. degree at Chonbuk National University and is also an assistant researcher at the WeGAT Research Center. His research interests include the development of voltage control methods for wind power plants.

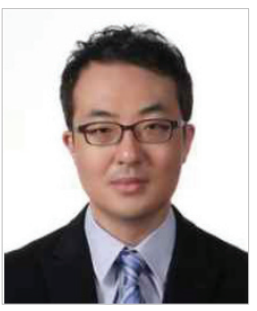

Hur Don He received B.S., M.S., and Ph.D. degrees in Electrical Engineering from Seoul National University in 1997, 1999, and 2004, respectively. His industry experience includes an internship in 2001 at Burns \& McDonnell Engineering Company, Kansas City, MO, USA. After finishing his Ph.D., he spent some time as a post-doctoral research associate at the Engineering Research Institute of Seoul National University and the University of Texas at Austin, TX, USA. $\mathrm{He}$ is currently an associate professor in the Department of Electrical Engineering at Kwangwoon University, Seoul, Korea, where he is affiliated with the power and energy systems area. He is a life member of KIEE and author or co-author of over 100 publications, studies, reports, and journal articles. His research interests relate broadly to modeling, analysis, and optimization of electric power and overall energy systems to feature the role and possible evolution of non-conventional energy resources, such as renewable generation and energy storage.

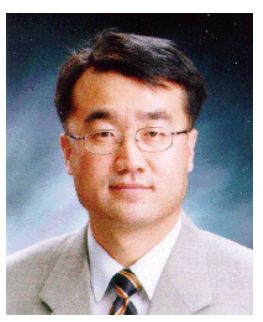

Yong Cheol Kang $\mathrm{He}$ received his B.S., M.S., and Ph.D. degrees from Seoul National University, Korea, in 1991, 1993, and 1997, respectively. He has been with Chonbuk National University, Korea, since 1999. He is currently Professor at Chonbuk National University, Korea, and the director of the WeGAT Research Center. He is also with the Smart Grid Research Center at Chonbuk National University. His research interests include the development of new protection and control systems for wind power plants and the enhancement of wind energy penetration levels. 